\begin{tabular}{ccc}
\hline & International Journal of Engineering \& Technology, $7(2.7)(2018) 249-252$ \\
SPC & International Journal of Engineering \& Technology \\
Website: $w w w . s c i e n c e p u b c o . c o m / i n d e x . p h p / I J E T$ & Research Paper \\
\hline
\end{tabular}

\title{
Speech enhancement using discrete kalmanfilter algorithm
}

\author{
J. Shanmukha ${ }^{1 *}$, K. S. Ramesh ${ }^{2}$, S. Koteswar Rao ${ }^{3}$ \\ ${ }^{1}$ M.TECH Student ,K. L. E. F, Vaddeswaram, Guntur \\ ${ }^{2}$ Prof. Department of E. C. E, K. L. E. F, Vaddeswaram, Guntur \\ ${ }^{3}$ Prof. Department of E. C. E, K. L. E. F, Vaddeswaram, Guntur \\ *Corresponding author E-mail: iamshnmk@gmail.com
}

\begin{abstract}
Enhancement of speech deals with the noise reduction which is mostly essential in many applications like teleconferencing sys tem. The nearness of added substance foundation noise degenerate the discourse in correspondence condition. In the event that the commotion is developing slower than discourse which implies noise is more stationary than speech .Estimation of noise ends up noticeably s impler when delays happened in speech. In this work discrete Kalman channel calculation which gauges the inward condition of a direct powe r-ful framework.
\end{abstract}

Keywords: Kalman Filter; Speech Enhancement; LPC; Wiener Filter.

\section{Introduction}

Most of the interactions between humans are happened via speech communication so there is a vast research happening over process engaged with creation and impression of discourse. The conduct of foundation clamor in discourse decreases the coherence of discourse. Cleaning of boisterous discourse is as yet a test to the region of flag preparing. The speech signs are measured by its quality and clarity intelligibility is a destination measure which pre-dicts the word rate that might be understandable by audience [1-4].Enhancement is improvement in quality of nature of something,related to speech enhancement means change /improving the understandability. Two vital criteria are chiefly utilized for execu-tion those are quality and intelligibility Enhancing of discourse debased by commotion, is the most essential field of discourse The essential goal of numerous Speech Enhancement calculations is to enhance the perceptual nature of extricating discourse motion from loud discourse. Expelling commotion from loud discourse is testing issue on the grounds that a ghastly property of non-stationary clamor is extremely hard to assess and foresee. Dis-course improvement has a different applications like ATM ma-chines and PCs, video chatting frameworks, biomedical flag pre-paring, portable amplifiers, discourse acknowledgment based se-curity gadgets

\section{Speech enhancement methods}

Various speech enhancement methods are available at present let us discuss a few methods and their drawbacks

\subsection{Weiner filter}

Weiner filter is one of the most widely used algorithms in speech enhancement study, if both speech signal and noise signal esti-mates are exactly true, Weiner filter gives the optimal estimate of the clean signal. It involves linear estimation of required /desired signal by modifying the weights ,mean squared error decreased between [6]the required /desired signal and filter output .

$H(\varphi)=\left[\frac{|\hat{S}(\varphi)|^{2}}{|\hat{S}(\varphi)|^{2}+|\hat{N}(\varphi)|^{2}}\right]$

$$
\widehat{S}(\varphi)=H(\varphi) S(\varphi)
$$

$\mathrm{H}$ - Stands for wiener filter

$\mathrm{S}-$ Stands for corrupted speech

$\mathrm{N}$ - Stands for noise spectra

Wiener filter has a zero phase spectrum, this is similar to spectral subtraction algorithm in this filter, and filter assumes that noise and signal of interest are ergodic and not correlated to each other, to assume stationary the signal is broken into frames to accommo-date the non-stationary of speech signal and this process is com-monly [5] [6]done in speech processing research.

Generalization of wiener filter is found by giving a noise correlation constant and power constant to filter.

$H(\varphi)=\left[\frac{|\hat{S}(\varphi)|^{2}}{|\hat{S}(\varphi)|^{2}+|\hat{N}(\varphi)|^{2}}\right]$

\subsection{Limitations of wiener filter}

The real restriction of wiener sifting preparing is mutilations happened by the commotion range the explanations behind those twists of immediate estimation of extent are limited fluctuation of the clamor control range ,cross item terms in the conditions, ineffectively outlined wiener channel comes about a low apparent quality and lesser data content [6] [7], than the first discourse flag. It is hard to assess both greatness and stage in a satisfactory tradeoff in calculation utilizing loud stage in light of here and now size estimation. 


\subsection{Linear predictive coding (L.P.C)}

Linear predictive analysis is a powerful tool using in speech enhancement, speech oriented research .LPC provides accurate estimates of speech and its relative speed in computation .LPC starts with supposition in which the speech signal is prepared by buzz. Estimating the intensity and frequency of the remaining buzz and the process of removing of formants is known as inverse filtering LPC is majorly used for data reduction applications the major idea of LPC is that every sample of speech signal represented in linear equations of previous specimens (inputs and outputs) and we use LPC to estimate those coefficients.s.

\section{Kalman filter}

\subsection{Discrete kalman filter}

Kalman channel is a standout amongst the most vital ideal channels to evaluate the condition of dynamic framework from arrangement of commotion estimation and resolve a blunder which is contained in discourse, in the wake of going it through a mutilated channel. For this, the scientist found that the Kalman channel could be utilized to illuminate and smother the moderate calculations of the Wiener channel, which is additionally one of the normal versatile separating strategies connected to discourse. In view of this discovering, it was thusly presumed that the Kal-man separating has an exact estimation and great developing strat-egy for discourse. It incorporates for the most part two purposes: estimation and execution investigation of estimator. Kalman sepa-rating incorporates principally two purposes: estimation and exe-cution examination of estimator. As it utilizes an entire portrayal of the likelihood of its estimation mistakes the "plan parameters" of the accompanying estimation frameworks are

- Types of sensors to be utilized;

- Locations and introductions of the different sensor write regarding the framework to be evaluated

- Allowable clamor qualities of the sensors

- Pre-separating techniques for smoothing sensor commotion

- Data inspecting rates for the different sensor composes and

- The level of model rearrangements

Let $a(n)$ and $b(n)$ be the set of observations and the pro- cess to be estimated. These are given by

$\mathrm{A}(\mathrm{n})=\mathrm{b}(\mathrm{n})+\mathrm{v}(\mathrm{n})$

Let $b(n)$ be an AR (1) process of the form

$$
b(n)=y(1) x(n-1)+w(n)
$$

$w(n)$ And $v(n)$ are uncorrelated

$$
\hat{b}(n)=y(1) \hat{b}(n-1)+K[a(n)-y(1) \hat{b}(n-1)]
$$

Where $K$ is a constant-Kalman gain which minimizes the meansquare error $E\left\{|b(n)-\overleftarrow{b}(n)|^{2}\right\}$,

If we let $\mathbf{B}(n)$ be the p-dimensional state vector

$$
\mathbf{B}(n)=\left[\begin{array}{c}
b(n) \\
b(n-1) \\
\vdots \\
b(n-p+1)
\end{array}\right]
$$

$\mathbf{B}(n)=\left[\begin{array}{ccccc}a(1) & a(2) & \cdots & a(p-1) & a(p) \\ 1 & 0 & \cdots & 0 & 0 \\ 0 & 1 & \cdots & 0 & 0 \\ \vdots & \vdots & \cdots & & \vdots \\ 0 & 0 & \cdots & 1 & 0\end{array}\right] \mathbf{B}(n-1)+\left[\begin{array}{c}1 \\ 0 \\ 0 \\ \vdots \\ 0\end{array}\right] w(n)$

And

$a(n)=\left[\begin{array}{llll}1 & 0 & \cdots & 0\end{array}\right] \mathbf{B}(n)+v(n)$

Using matrix notation to simplify these equations we have

$\mathbf{B}(n)=\mathbf{R B}(n-1)+\mathbf{w}(n)$

$y(n)=\mathbf{c}^{\mathrm{T}} \mathbf{x}(n)+v(n)$

Where $\mathrm{R}$ is a $p \times p$ state transition matrix, $\mathbf{w}(n)=\left[\begin{array}{llll}w(n) & 0 & \cdots & 0\end{array}\right]^{T}$ is a vector noise process, and $\mathrm{c}$ is a unit vector of length $p$

$\hat{\mathbf{B}}(n)=\mathbf{R} \hat{\mathbf{B}}(n-1)+\mathbf{K}\left[a(n)-\mathbf{c}^{\mathrm{T}} \mathbf{R} \hat{\mathbf{B}}(n-1)\right]$

$\mathrm{K}$ is a Kalman gain, Eq. (10) may be easily general to non-stationary processes Let $\mathbf{B}(n)$ be a state vector of dimension $p$

$\mathbf{B}(n)=\mathbf{R}(n-1) B(n-1)+\mathbf{w}(n)$

Simplifying eq (11)

$\hat{B}(n)=R(n-1) \hat{\mathbf{x}}(n-1)+\mathbf{K}(n)[a(n)-\mathbf{C}(n) R(n-1) \hat{B}(n-1)]$

$\mathbf{A}(n), \mathbf{C}(n), \mathbf{Q}_{w}(n)$, and $\mathbf{Q}(n)$ Are known.

\subsection{Purpose of kalman filter}

Proposed Kalman sifting strategy which takes into considera-tion stationary and non-stationary of discourse and at same time it is fit to evaluating blunders exact more than another channels. Kalman Filter is a versatile minimum square blunder channel that gives a proficient computational recursive answer for assessing a flag in nearness of Gaussian commotions to ceaselessly refresh the best gauge of the framework's present state. Additional than 20 years have passed kalman's unique paper is as yet managing nu-merous daily papers, applications an variety in light of the first of Kalman channel. It has withstood the trial of time!

In the phase of estimation, the estimations of some obscure parameters are assessed past to the operation of the channel. For example, the change of estimation clamor can be evaluated from some disconnected example estimations. The fluctuation of the procedure clamor is more heuristic and tuning should be possible to change its underlying incentive to show signs of improvement precision of model. The estimations of different parameters are thought to be known either from the laws of material science or from other intelligent suspicion.

\subsection{Implementation of kalman filter}

Once the estimations of the parameters are distinguished, the exactness of the evaluated model will be tried in the model avoca-tion organize. A progression of state expectations is assessed through the time refresh and estimation refresh conditions (circle operation of Kalman channel). The accuracy of these forecasts is contrasted next with the genuine perceptions and residuals are computed. In the event that the estimations of the residuals are too enormous (inadmissible), calibrating is finished by changing the estimations of the parameters R, Q (estimation and process clamor separately) of 
the model until the point that a worthy remaining level is accomplished. Accepting A, H, R and Q are static, a Mat lab and R programming are composed to locate the best esteems for these parameters that would deliver the assessed Xк (created by those parameters) are contrasted with the genuine time ar-rangement. The range for $\mathrm{An}$ and $\mathrm{H}$ is limited in the vicinity of 0 and 2 since these two scalars that should convey positive relation-ship. The augmentation for the estimations of $\mathrm{An}$ and $\mathrm{H}$ is 0.1 for each progression. The difference of the time arrangement infor-mation can be computed disconnected and the range for $\mathrm{R}$ and $\mathrm{Q}$ is confined in the vicinity of 0 and the fluctuation of $\mathrm{t}$ he infor-mation. The estimations of $\mathrm{R}$ and $\mathrm{Q}$ are increased by 1 in each progression. The underlying esteems decided for $\mathrm{x}^{\wedge}-\mathrm{k}$ and P-k are normal 0 and 1 individually. The jobs needing to be done is to discover the estimations of these parameters and decide the best gauge of condition of discourse scientifically, in addition to dis-cover the estimator with the littlest conceivable blunder change.

\section{Simulation and results}

In this paper the stimulation source code is written in MATLAB 17 to employ Discrete Kalman filter in speech enhancement .The main reason for using MATLAB, it is most influential program-ming language for matrices, generally signal noise is an array of numbers so MATLAB be a very practical approach. In this paper we considered a sample speech signal and white noise of variance 0.001

We generated the original speech signal and corrupted speech signal with white noise, by using the Discrete Kalman filter we reconstructed the original signal from the corrupted speech signal The following figures shows the original speech signal ,figure 2 shows the corrupted or noisy speech signal, figure 3 shows the estimated speech signal, figure 4 shows the cross correlation be-tween the original signal and noisy speech signal figure 5 shows the cross covariance between the original speech signal and esti-mated speech signal

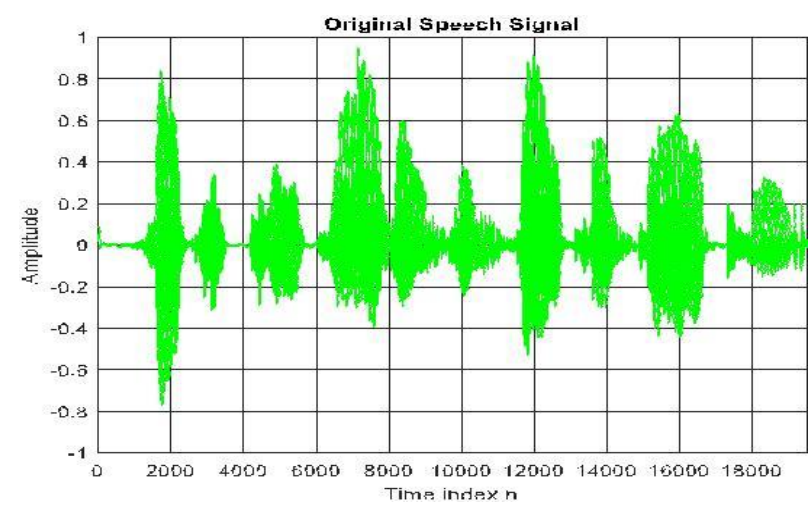

Fig. 1:

In the above figure we have considered a speech signal of frequency $10 \mathrm{~K}$ signal and read with the help of command wave read in matlab

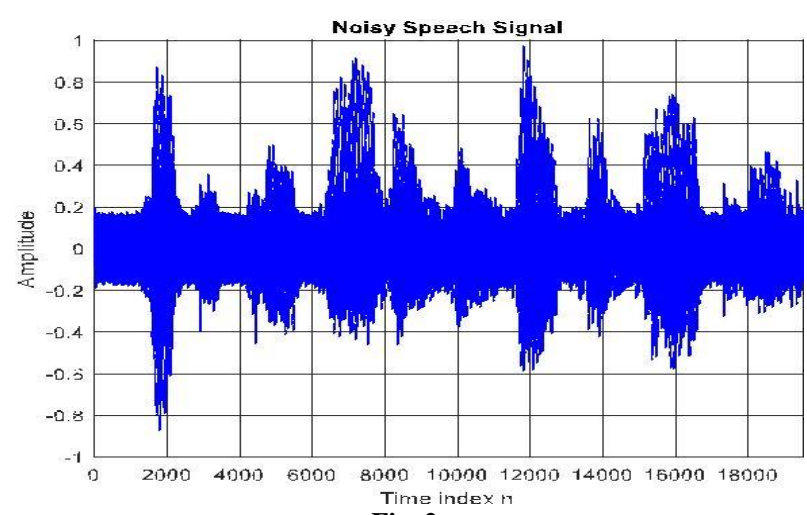

Fig. 2:
Now we generated the white Gaussian noise of variance 0.001 , which corrupts the original signal

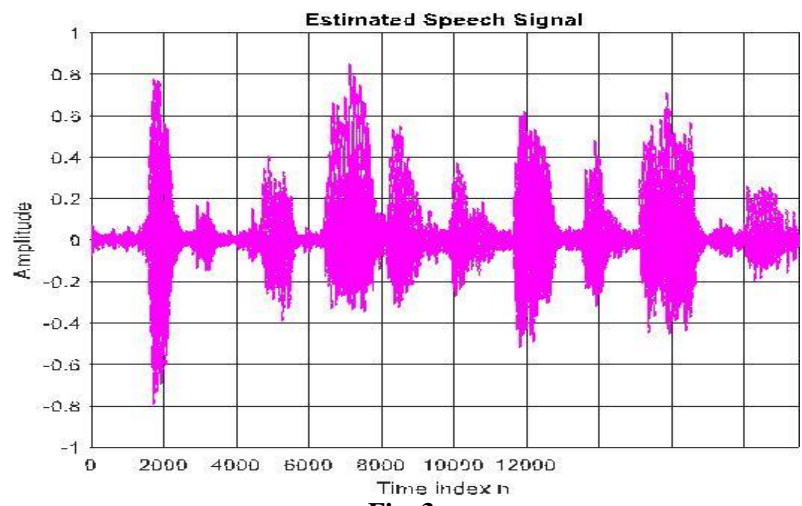

Fig. 3:

The corrupted speech signal is given to the filter to extract the original signal through estimates by detecting the noise signal and eliminating the noise

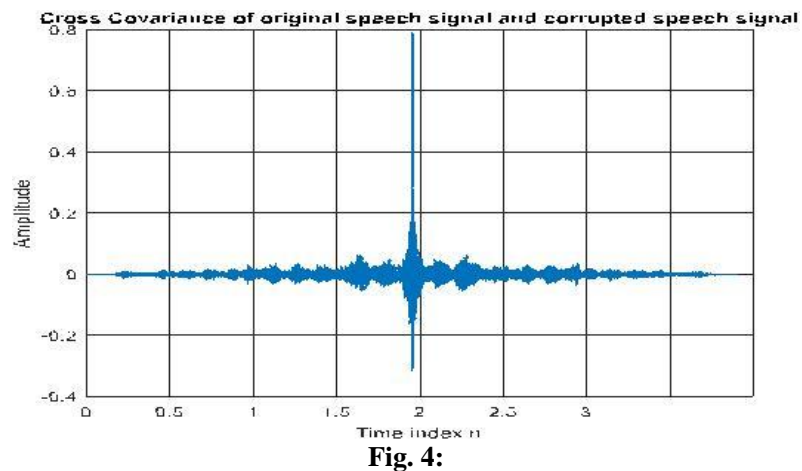

We are finding the cross covariance between the original signal and the corrupted speech signal

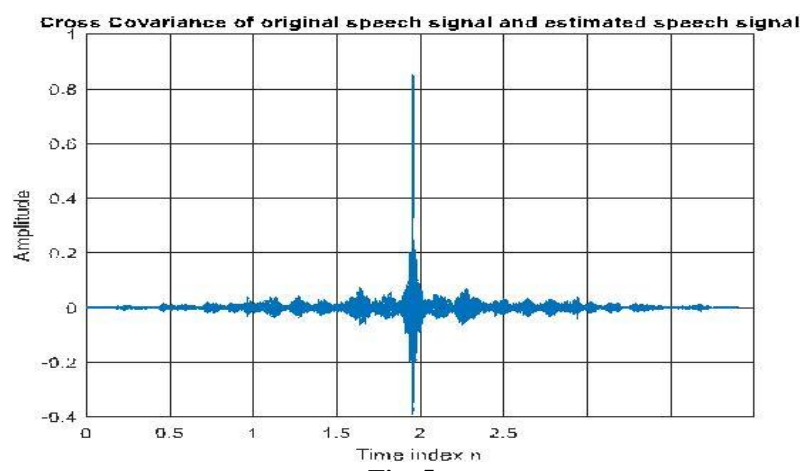

Fig. 5

Now we are calculating the cross covariance among the original and corrupted speech signal and we observed that both are identical which means there is improvement in the intelligibility In Kalman filter while estimating the results in figures be-fore, Kalman filter needs some time to before stable to estimate the reconstructed speech output, both $\mathrm{Q}$ and $\mathrm{R}$ have to be identical to the original signal Cross Covariance (OR) Cross Correlation is mostly used for measuring the similarities among the given two signals, based on the amount of similar parts to prediction and comparing the differences among two signals

\section{Conclusion}

In this paper Discrete Kalman filter is implement and enhance-ment of the speech signal by using MATLAB (2017) software .the results shown had been obtained by testing the sample speech signal to provide optimal performance by improving the intelligi-bility, cross 
covariance had been performed during the stimulation, the work done is doing well to achieve the solution for stated problem. Hence the Discrete Kalman filter algorithm is most effi-cient for speech enhancement.

\section{References}

[1] Cho JH, Chang SA, Kwon HS, Choi YH, KoSH, Moon SD, Yoo SJ, Song KH, Son HS, Kim HS, Lee WC, Cha BY, Son HY \& Yoon KH (2006), Long-term effect of the internet-based glucose monitoring system on HbA1c Reduction and glucose stability: a 30-month follow-up study for diabetes management with a ubiquitous medical care system. Diabetes Care 29, 2625-2631.

[2] Fauci AS, Braunwald E, Kasper DL \& Hauser SL (2008), Principles of Harrison's Internal Medicine, Vol. 9, 17thedn. McGraw-Hill, New York, NY, pp.2275-2304.

[3] Kim HS \& Jeong HS (2007), A nurse short message service by cellular phone in type-2 diabetic patients for six months. Journal of Clinical Nursing 16, 1082-1087.

[4] Lee JR, Kim SA, Yoo JW \& Kang YK (2007), The present status of diabetes education and the role recognition as a diabetes educator of nurses in korea. Diabetes Research and Clinical Practice 77, 199204.

[5] McMahon GT, Gomes HE, Hohne SH, Hu TM, Levine BA \& Conlin PR (2005), Web-based care management in patients with poorly controlled diabetes. Diabetes Care 28, 1624-1629.

[6] Thakurdesai PA, Kole PL \& Pareek RP (2004), Evaluation of the quality and contents of diabetes mellitus patient education on Internet. Patient Education and Counseling 53, 309-313. 